L'avènement des lasers

femtoseconde Térawatts a ouvert de nouvelles perspectives quant à l'utilisation des lasers pour sonder l'atmosphère. En particulier, le mode de propagation sous forme de filaments, comparable à un " laser blanc ", a permis le développement du LIDAR à lumière blanche dans le cadre de la collaboration Téramobile. Un autre volet d'applications réside dans l'utilisation de la colonne de

plasma générée au sein des filaments, qui est plus conductrice que l'air, pour déclencher et guider la foudre.

Figure 1. Filament de lumière blanche généré dans l'atmosphère par un laser infrarouge ultrabref

\title{
Téramobile : un laser femtoseconde pour étudier l'atmosphère
}

Guillaume Méjean ${ }^{1}$, Jérôme Kasparian, Jean-Pierre Wolf

Téramobile, LASIM UMR CNRS 5579, Université Lyon 1, 69622 Villeurbanne Cedex, ${ }^{1}$ Lauréat du prix “Jeune Chercheur” St Gobain 2005 de la SFP

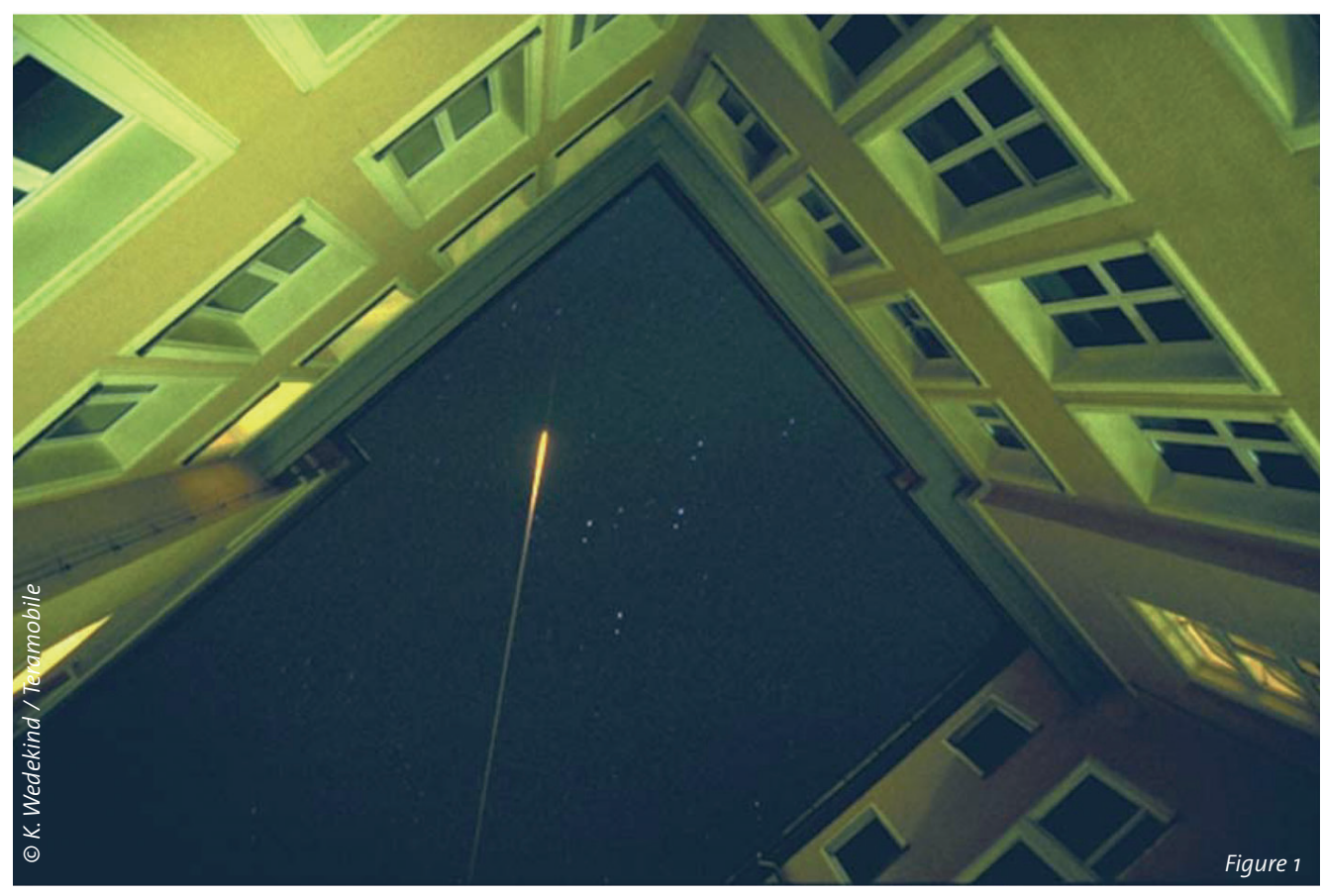

\section{Introduction}

La caractérisation à distance de l'atmosphère est nécessaire pour déterminer sa composition chimique et pour étudier les processus dynamiques comme le réchauffement global, le trou d'ozone, la pollution urbaine ou les prévisions météorologiques. La spectroscopie infrarouge à transformée de Fourier (FTIR, pour Fourier-Transform InfraRed spectroscopy) ou la spectroscopie optique d'absorption différentielle (DOAS, Differential Optical Absorption Spectroscopy) mesurent les concentrations de vastes groupes de constituants atmosphériques, en utilisant l'absorption de la lumière du soleil ou de la lune sur son chemin à travers l'atmosphère [1]. Mais ce chemin est fixé par la position de la source lumineuse utilisée. Au contraire, le LIDAR (LIght Detection And Ranging, un analogue optique du radar, voir encadré p.11) utilise la rétrodiffusion par l'air de la lumière émise par un laser impulsionnel [1]. Le rayon laser pouvant être orienté, il n'a pas un chemin prédéterminé, ce qui lui permet de fournir des cartes tridimensionnelles de concentration de l'atmosphère en traces de gaz ( $\mathrm{NO}, \mathrm{NO}_{2}, \mathrm{SO}_{2}$, benzène, toluène, ozone...) ou d'aérosols (comme les suies issues de combustion ou du trafic automobile, ou les nuages stratosphériques polaires impliqués dans la destruction de la couche d'ozone). Cependant, le LIDAR est généralement limité à la mesure d'une espèce à la fois.

La technique de mesure idéale combinerait le large spectre du DOAS et du FTIR, avec la résolution spatiale du LIDAR. Elle permettrait par ailleurs une mesure à distance des aérosols, naturels ou artificiels, sans l'apport d'informations extérieures provenant de ballons sondes, ou d'hypothèses a priori sur l'atmosphère, contrairement aux techniques actuelles.

La génération de lumière blanche [2,3], issue de la propagation d'un laser femtoseconde focalisé, semble être l'outil idéal. En effet, des progrès récents dans le domaine des lasers ultrabrefs ont montré que la "filamentation » (définie ci-dessous) des impulsions laser de forte puissance peut créer des régions de très forte intensité à de grandes distances. Ce processus apparaît lorsque la puissance du faisceau atteint ou dépasse une puissance dite puissance critique $\left(\mathrm{P}_{\text {crit }}=(1,22 \lambda)^{2} / 12,8 \mathrm{n}_{2}\right.$, soit quelques $\mathrm{GW}$ dans l'air), $\mathrm{n}_{2}$ représentant l'indice non linéaire de l'air (c'est-à-dire le facteur défini de manière à ce que l'indice de l'air s'écrive $\mathrm{n}=\mathrm{n}_{0}+\mathrm{n}_{2} \mathrm{I}$, I étant l'intensité incidente). Le profil transverse d'intensité du faisceau est quasi-gaussien ; il en est donc de même du profil d'indice. 
Le chemin optique à parcourir est donc plus long au centre de l'impulsion que sur ses bords. Le faisceau crée ainsi sa propre lentille convergente, dite lentille de Kerr, qui focalise le faisceau sur son axe. C'est ce qu'on appelle l'auto-focalisation par effet Kerr. En raison de cette auto-focalisation, l'intensité augmente jusqu'à générer un plasma. Le plasma contribue négativement à l'indice de réfraction, ce qui conduit à un équilibre entre l'effet Kerr qui focalise le faisceau et le plasma ainsi que la diffraction naturelle qui le défocalisent. Il se crée ainsi des structures autoguidées qu'on appelle filaments. Ces filaments peuvent se propager sur des distances pouvant dépasser la centaine de mètres, soit plusieurs ordres de grandeur au-delà de la distance de Rayleigh, distance pour laquelle le profil transverse d'un faisceau se propageant linéairement est affecté par la diffraction.

Un analogue de l'effet Kerr se manifeste aussi dans l'espace temporel. En effet, l'indice de réfraction non-linéaire dans les ailes de l'impulsion (au début et à la fin de l'impulsion), où l'intensité est plus faible, n'est pas le même qu'au sommet de l'impulsion où l'intensité est grande. Il se produit alors un déphasage dépendant du temps : l'automodulation de phase (en anglais self phase modulation, ou SPM). La valeur instantanée de la pulsation de l'impulsion étant égale à la dérivée de la phase, cela se traduit par la génération de nouvelles fréquences $\omega$ sur le front montant $(\Delta \omega<0)$ et sur le front descendant $(\Delta \omega>0)$ de l'impulsion, comme le traduisent les équations ci-dessous écrites dans le référentiel attaché à l'impulsion, où $\phi$ représente la phase de l'impulsion, $\Delta \omega$ la pulsation propre de l'impulsion, c la vitesse de la lumière et z l'axe de propagation :

$\phi(\mathrm{t})=\omega_{0} \mathrm{t}-\mathrm{z} \cdot \omega_{0} / \mathrm{c} \cdot \mathrm{n}(\mathrm{t})=\omega_{0} \mathrm{t}-\mathrm{z} \cdot \omega_{0} / \mathrm{c} \cdot\left(\mathrm{n}_{0}-\mathrm{n}_{2} \mathrm{I}(\mathrm{t})\right)$ $\omega(\mathrm{t})=\mathrm{d} \phi / \mathrm{dt}=\omega_{0}-\mathrm{z} \cdot \omega_{0} / \mathrm{c} \cdot \mathrm{n}_{2} \cdot \mathrm{dI} / \mathrm{dt}$
La haute intensité dans les filaments (4 à $6 \mathrm{x}$ $\left.10^{13} \mathrm{~W} / \mathrm{cm}^{2}\right)$ génère une importante automodulation de phase et donc un large continuum de lumière blanche s'étendant de $230 \mathrm{~nm}$ à $4,5 \mu \mathrm{m}$. Cette émission cohérente de lumière blanche est clairement visible à l'œil nu (voir figure 1) [2], bien que le laser utilisé émette dans l'infrarouge, et est émise essentiellement vers l'avant ; mais une part significative en est également émise vers l'arrière, ce qui est particulièrement favorable au LIDAR.

Ce nouveau mode de propagation nonlinéaire et ses perspectives d'applications à la télédétection atmosphérique sont à l'origine $\mathrm{du}$ projet Téramobile $[4,5]$. Ce projet francoallemand, cofinancé par le CNRS et la $\mathrm{DFG}^{(1)}$, vise en particulier à démontrer la faisabilité d'un LIDAR multi-composants basé sur des impulsions laser ultrabrèves, pour la mesure tant des polluants gazeux que des aérosols atmosphériques. Le but est d'utiliser la propagation hautement non-linéaire de ce type de laser pour, soit utiliser le laser « blanc » pour un lidar à lumière blanche, soit délivrer des hautes intensités à distance de manière à induire in situ des effets non linéaires. Par ailleurs, le plasma généré dans les filaments pourrait permettre de déclencher et de guider la foudre.

A cet effet, la collaboration Téramobile a développé le premier laser Térawatt mobile. Il fournit des impulsions de $350 \mathrm{~mJ}$ en 70 femtosecondes, soit une puissance crête de $5 \mathrm{TW}$, à une longueur d'onde de $800 \mathrm{~nm}$ et à un taux de répétition de $10 \mathrm{~Hz}$ [6]. Outre le laser et sa suspension mécanique, le conteneur contient un laboratoire d'optique complet, y compris le conditionnement d'air, les alimentations électriques et un système de détection LIDAR.
Les filaments permettent de délivrer des hautes intensités à distance de manière à induire des effets non linéaires

in situ.
(1) Deutschen ForschungsGemeinschaft: organisme de financement de la recherche publique et universitaire en Allemagne.

\section{Le LIDAR}

La technique LIDAR consiste à envoyer des impulsions lasers dans l'atmosphère et à mesurer au niveau du laser la lumière rétrodiffusée en fonction du temps. Le faisceau laser, au cours de sa propagation, est absorbé et diffusé par les gaz et les aérosols de l'atmosphère. Une partie de cette lumière est rétrodiffusée vers l'émetteur. Pour collecter cette lumière, un télescope est placé colinéairement au laser et un détecteur avec une résolution temporelle est placé au foyer du télescope.

L'intérêt de cette technique est qu'elle est résolue spatialement grâce au temps de parcours de la lumière. L'intensité lumineuse rétrodiffusée I(z) nous renseigne sur la composition de l'atmosphère à la distance $z$ du laser.

Le LIDAR à lumière blanche utilise toutes les longueurs d'onde disponibles dans le spectre d'un faisceau laser térawatt femtoseconde, grâce à la génération de lumière blanche. À cet effet, il faut déterminer le signal rétrodiffusé en fonction du temps comme pour le LIDAR classique, mais aussi en fonction de la longueur d'onde. Le détecteur résolu en temps d'un LIDAR classique (un photomultiplicateur) est donc remplacé par un spectrophotomètre résolu en temps (spectromètre suivi d'un photomultiplicateur). Une autre variante du LIDAR est d'induire des effets non-linéaires à distance sur des cibles à détecter et de les identifier, en profitant de la capacité des filaments à transporter de hautes intensités à distance. 

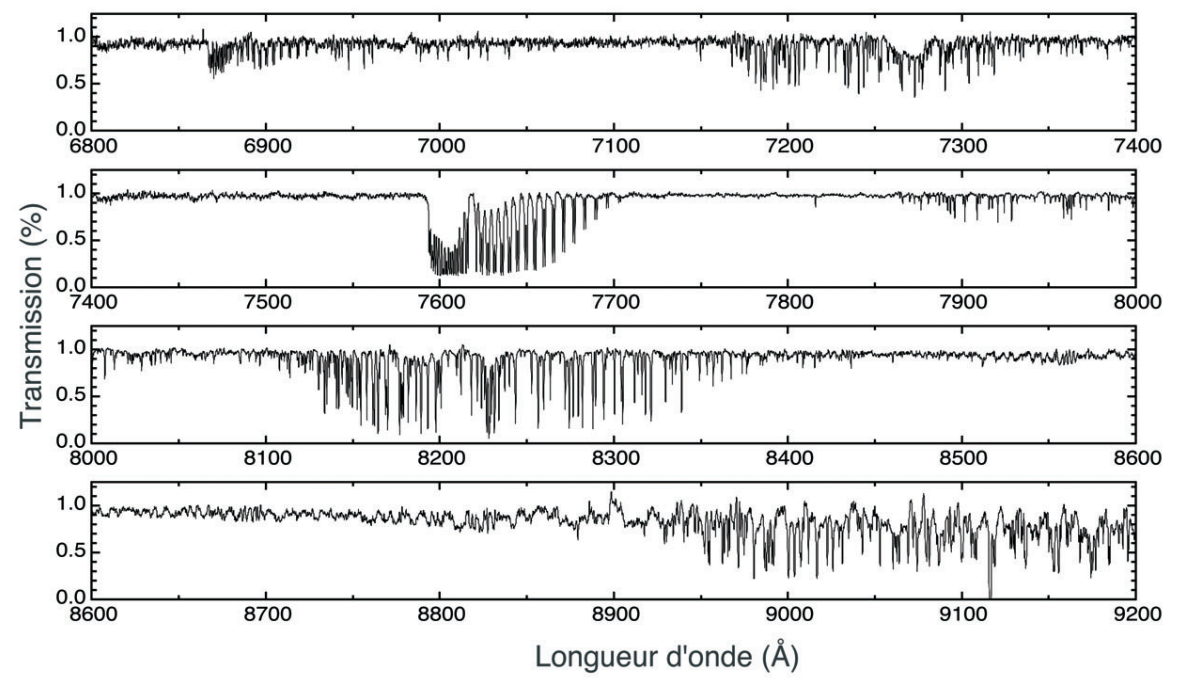

Figure 2. Spectre large bande à haute résolution de la transmission atmosphérique mesurée par LIDAR à lumière blanche. Ony reconnait les bandes d'absorption de l'oxygène (687-696 et 759-770 $\mathrm{nm}$ ) et de la vapeur d'eau (716-732, 811-853 et 893-925 nm). Ces absorptions permettent de déterminer la température et l'humidité de l'air, donc son humidité relative.

(2) Le filament créé à la base du nuage peut être considéré comme la source de lumière blanche.

Figure 3. Télédétection d'aérosols biologiques. Un nuage d'eau (en haut) ne produit pas de fluorescence, contrairement à un simulant biologique (en bas). Cette fluorescence, détectée en fonction de la distance et de la longueur d'onde, permet d'identifier et de localiser le nuage potentiellement dangereux.

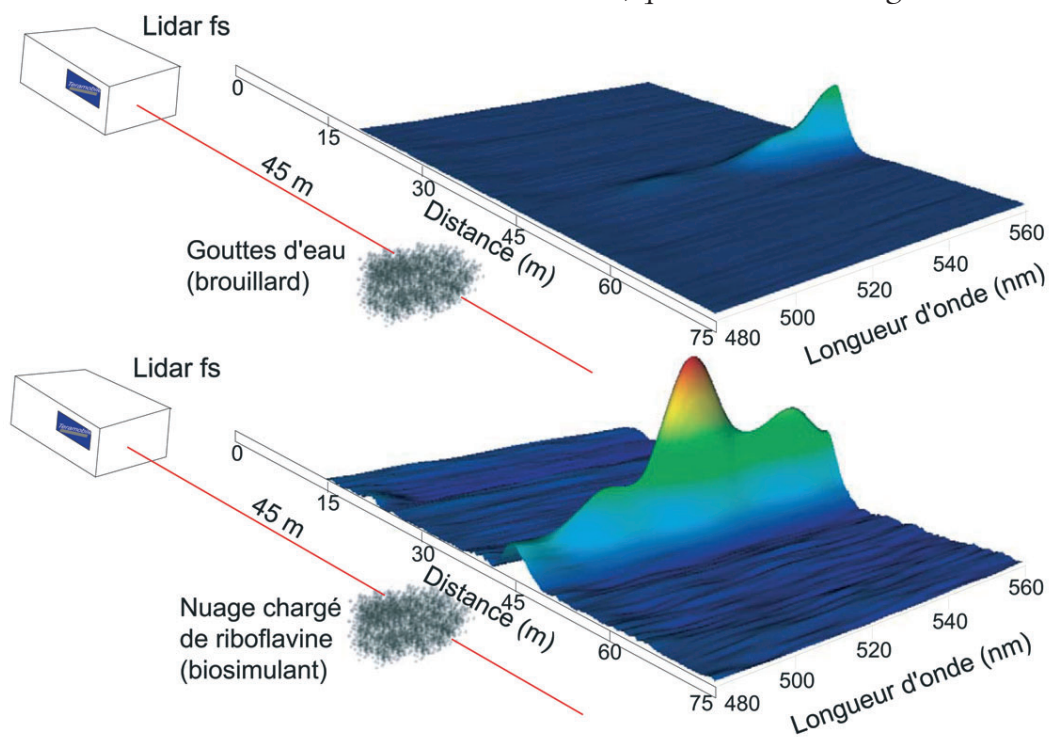

des bandes de l'oxygène, et d'autre part la teneur en vapeur d'eau, qui modifie l'absorption des raies de l'eau. Ceci permet de déterminer simultanément ces deux paramètres atmosphériques, et par conséquent d'en déduire un profil avec l'altitude de température et d'humidité relative, données précieuses pour la météorologie autant que pour la climatologie.

Parallèlement, la mesure du profil du halo rétrodiffusé par le nuage permet de caractériser la diffusion multiple du faisceau dans le nuage et de déterminer ainsi la distribution de taille des gouttes d'eau qui le constituent ; ceci ouvre la voie vers une caractérisation simultanée des nuages et des conditions thermodynamiques à leur voisinage : ces données permettront de mieux caractériser la formation des nuages et la croissance des gouttes d'eau en leur sein.

\section{Lidar multiparamètres à lumière blanche}

La possibilité de réaliser des mesures LIDAR à haute résolution sur une large bande spectrale est illustrée par la figure 2.A l'aide d'un télescope astronomique situé à proximité du laser, on mesure la transmission atmosphérique du faisceau rétrodiffusé par la base d'un nuage à $4,5 \mathrm{~km}$ de distance $^{(2)}$. On y reconnait la signature spectrale à haute résolution $(0,01 \mathrm{~nm})$ de l'atmosphère sur ce chemin d'absorption exceptionnellement long. Dans cette région spectrale, les principales bandes d'absorption observées sont celles de l'oxygène (687-696 et 759-770 $\mathrm{nm}$ ) et de la vapeur d'eau (716-732, 811-853 et 893-925 nm). Des simulations basées sur la base de données HITRAN [7] reproduisent parfaitement ce spectre. Ces simulations ont pour paramètres libres d'une part la température et sa variation avec l'altitude, qui fait varier la largeur des raies à mesurer. De plus, aucune technique à ce jour ne permet une analyse chimique à distance des aérosols. La forte puissance crête des impulsions femtoseconde offre l'opportunité d'arriver à ce résultat grâce à la génération in-situ d'effets nonlinéaires directement à l'intérieur des particules d'aérosols, comme la fluorescence pompée à plusieurs photons. Cette signature pourrait permettre d'identifier la composition chimique de la particule distante.

Nous avons récemment appliqué cette technique sur le terrain pour détecter à distance la pollution de particules d'eau par un traceur caractéristique des particules biologiques. Dans cette expérience, un nuage était produit dans une enceinte placée à une distance de $45 \mathrm{~m}$ du système Téramobile. Lorsque les gouttes de ce nuage sont dopées par de la riboflavine, utilisée comme biosimulant, la signature de la fluorescence 
excitée à deux photons au cœur des gouttelettes est visible sur le signal LIDAR. Au contraire, cette signature disparaît lorsque le nuage est composé d'eau pure (figure 3). Ce résultat démontre la possibilité d'utiliser le LIDAR pour distinguer des particules biologiques des aérosols naturellement présents dans l'atmosphère, bien que les particules aient des tailles identiques. Des simulations montrent que cette technique de LIDAR non linéaire va présenter un intérêt à longue distance. En effet, l'excitation linéaire nécessite un laser vers $256 \mathrm{~nm}$ : à cette longueur d'onde, la diffusion par les molécules de l'air est considérable, de sorte que le faisceau sera fortement atténué lors de sa propagation dans l'air. De plus, il est possible de mettre en forme temporellement les impulsions femtosecondes, et d'éloigner ainsi la distance de détection tout en accroissant la sélectivité de la méthode.

\section{Vers un paratonnerre laser ?}

L'ionisation de l'air dans les filaments ouvre également un autre volet d'application atmosphérique : la mise au point d'un paratonnerre laser. L'idée de déclencher la foudre avec des lasers est presque aussi ancienne que les lasers eux-mêmes. Un paratonnerre laser permettrait en effet d'assurer la protection de sites sensibles tels que des centrales électriques et des aéroports. Dans les années 1970 et 1980, les lasers nanoseconde ont montré leurs limites, ne parvenant pas à générer dans l'air un canal conducteur suffisamment long. Mais les filaments autoguidés produits par les lasers femtoseconde-terawatt, qui présentent une densité d'électrons supérieure à celle requise pour déclencher une décharge électrique dans l'atmosphère, soit $5.10^{11} \mathrm{~cm}^{-3}$, ont ravivé l'intérêt pour le sujet. Nous avons récemment déclenché et guidé des décharges de haute tension en laboratoire grâce à des filaments ionisés générés par un laser femtoseconde. Grâce à sa mobilité, le laser Téramobile a été placé dans un laboratoire équipé d'un générateur de haute tension (2 MV), et son faisceau a été aligné avec les électrodes. De la sorte, les filaments générés par le laser courtcircuitaient l'intervalle de 1 à 5 mètres entre les électrodes modélisant respectivement la terre et un nuage chargé. Les décharges, erratiques lorsque le laser était éteint, étaient guidées par les filaments lorsqu'ils étaient présents (figure 4) [8,9]. De plus, la tension de claquage était réduite d'environ $30 \%$ par rapport à la décharge libre. Nous avons ainsi déclenché des décharges à des tensions qui ne permettraient pas de décharge sans laser.

Ces résultats sont très encourageants quant à la possibilité d'utiliser les filaments pour contrôler la foudre. Ils ont récemment été complétés par
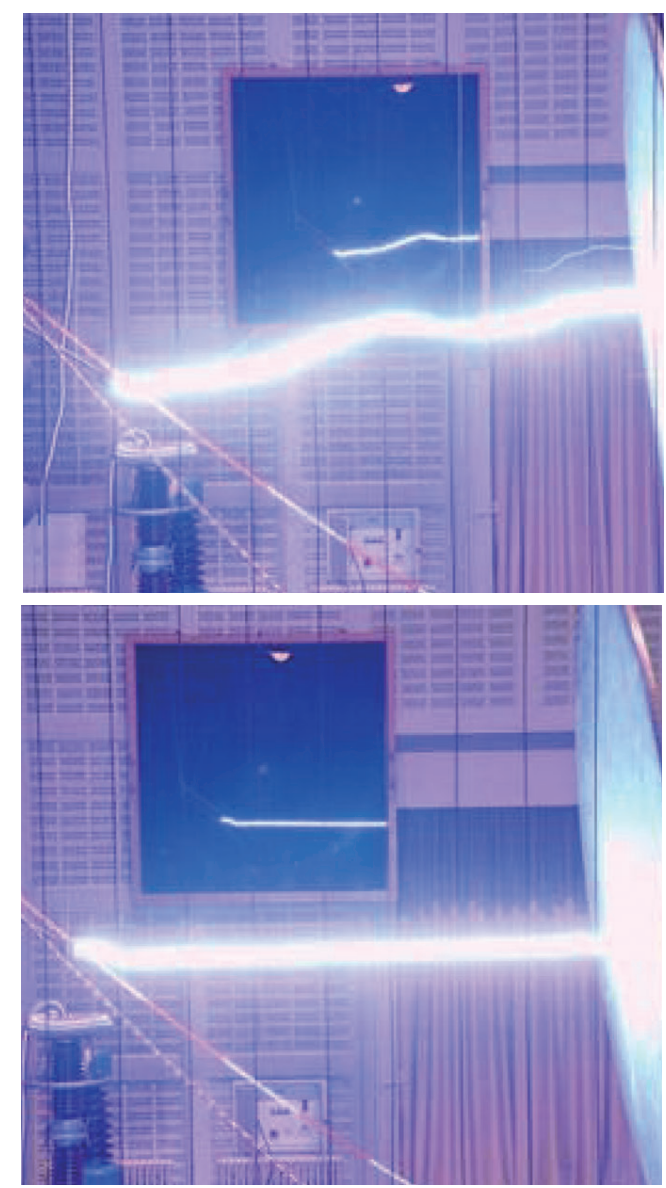

des simulations plus réalistes, lors desquelles les décharges ont été déclenchées dans des conditions comparables mais sous une pluie artificielle : grâce à la survie des filaments, mentionnée plus haut, l'efficacité de déclenchement et de guidage n'est que faiblement réduite. Si ces résultats portent à l'optimisme, des expériences complémentaires restent à mener pour déterminer si les mesures réalisées en laboratoire en échelle réduite peuvent être extrapolées à des distances de quelques centaines de mètres impliquant un mécanisme de décharge plus complexe. Par ailleurs, le coût d'un système comme le Téramobile le destine spécifiquement à la protection de sites sensibles ou au déclenchement d'éclairs pour le test de prototypes d'équipements dont on souhaite caractériser la résistance à la foudre.

\section{Conclusion}

Ces dernières années, la compréhension de la propagation non-linéaire des impulsions laser ultrabrèves et de forte puissance a progressé à grands pas. Ces progrès autorisent désormais les chercheurs à s'intéresser à des applications à l'atmosphère, telles que la télédétection de polluants ou le contrôle de foudre. Des résultats prometteurs ont été obtenus récemment, en particulier par l'équipe franco-allemande du projet Téramobile.
Figure 4. Décharge électrique haute tension (1 MV) libre (figure du haut), et guidée par des filaments générés par laser (figure du bas).

\section{Références}

[1] R. A. Meyers (Ed.), Encyclopedia of analytical chemistry, Vol. 3, Wiley, Chichester (2000).

[2] L. Wöste et al., Laser und Optoelektronik 29 (1997) ;

P. Rairoux et al., Remote sensing of the atmosphere using ultrashort laser pulses, Applied Physics B 71 (2000), 573-580.

[3] Lumière laser sur la chimie atmosphérique, La Recherche, septembre 2003

[4] J. Kasparian, et al., White-light filaments for atmospheric analysis, Science, 301 (2003), 61-64.

[5] http://www.teramobile.org

[6] J. Kasparian, Le projet Téramobile et ses applications, Photoniques 21, janvier-février 2006, p.28.

[7] http://www.hitran.com

[8] J. Kasparian, "Téramobile" lance ses éclairs, CNRS Thema, avril 2003.

[9] Le laser fait craquer la foudre, Sciences et Avenir, juillet 2001. 